\title{
Solutions for Toda systems on Riemann surfaces
}

\author{
JIAYU LI AND YUXIANG LI \\ Dedicated to Professor Ding Weiyue on the occasion of his 60th birthday
}

\begin{abstract}
In this paper we study the solutions of Toda systems on Riemann surface in the critical case, proving a sufficient condition for existence.
\end{abstract}

Mathematics Subject Classification (2000): $35 \mathrm{~J} 60$ (primary); 58G03, 35J45 (secondary).

\section{Introduction}

Let $(\Sigma, g)$ be a compact Riemann surface with unit area 1. Ding-Jost-Li-Wang [8] studied the differential equation $\Delta u=8 \pi-8 \pi h e^{u}$ on $(\Sigma, g)$, the so-called Kazdan-Warner problem [17] related to the Abelian Chern-Simons model (see [3, $4,6,5,7,1,2,9,10,11,23,13,14,24,21]$, etc). They pursued a variational approach to the problem, trying to minimize the functional

$$
J(u)=\frac{1}{2} \int_{\Sigma}|\nabla u|^{2} d V_{g}+8 \pi \int_{\Sigma} u d V_{g}-8 \pi \log \int_{\Sigma} h e^{u} d V_{g} \geq C, \quad \text { in } H^{1,2}(\Sigma)
$$

for some constant $C>0$. Because it is the critical case of the Moser-Trudinger inequality (1.1), the analysis is subtle.

Let $K$ denote the Cartan matrix for $\mathrm{SU}(N+1)$, i.e.

$$
K=\left(a_{i j}\right)=\left(\begin{array}{rrrrrr}
2 & -1 & 0 & \ldots & \ldots & 0 \\
-1 & 2 & -1 & 0 & . & 0 \\
0 & -1 & 2 & -1 & \ldots & 0 \\
\ldots & \ldots & \ldots & \ldots & \ldots & \ldots \\
0 & \ldots & \ldots & -1 & 2 & -1 \\
0 & \ldots & \ldots & 0 & -1 & 2
\end{array}\right)
$$

The research was supported by NSFC.

Pervenuto alla Redazione il 27 aprile 2005 e in forma definitiva il 2 novembre 2005. 
In this paper we consider the Toda systems on $(\Sigma, g)$ which are related to the nonAbelian Chern-Simons model [22]:

$$
-\Delta u_{i}=M_{i}\left(\frac{\exp \left(\sum_{j=1}^{N} a_{i j} u_{j}\right)}{\int_{\Sigma} \exp \left(\sum_{j=1}^{N} a_{i j} u_{j}\right)}-1\right), \quad \text { for } 1 \leq i \leq N .
$$

If $M_{i}<4 \pi$ Jost-Wang [16] proved the existence of solutions and in the case where $\Sigma$ is a torus, $N=2, \max \left\{M_{1}, M_{2}\right\}>4 \pi$ and $\min \left\{M_{1}, M_{2}\right\} \neq 4 \pi$, MarcelloMargherita [20] proved the same result. tional

They studied the problem by considering for $u_{1}, \ldots, u_{N} \in H^{1,2}(\Sigma)$ the func-

$$
\begin{aligned}
\Phi_{\left(M_{1}, \ldots, M_{N}\right)}\left(u_{1}, \ldots, u_{N}\right)= & \frac{1}{2} \sum_{i, j=1}^{N} \int_{\Sigma} a_{i j}\left(\nabla u_{i} \nabla u_{j}+2 M_{i} u_{j}\right) d V_{g} \\
& -\sum_{i=1}^{N} M_{i} \log \int_{\Sigma} \exp \left(\sum_{j=1}^{N} a_{i j} u_{j}\right) d V_{g} .
\end{aligned}
$$

Jost-Wang [16] proved that the functional has a lower bound if and only if

$$
M_{i} \leq 4 \pi, \quad \text { for } i=1,2, \ldots, N .
$$

Marcello-Margherita [20] obtained a non-minimizing critical point of the functional motivated by an earlier paper of Struwe-Tarantello [23]. The idea was later also used by Djadli and Malchiodi [12] to study the existence of conformal metrics with constant $Q$-curvature. It is clear that $M_{i}=4 \pi$ is the critical case of the functional. Whether it admits minimizer is subtle. In this paper we study this problem. For simplicity, we consider only the case that $N=2$, the general case need only more calculations. In our case the functional is

$$
\begin{aligned}
\Phi\left(u_{1}, u_{2}\right)= & \frac{1}{2} \sum_{i, j=1}^{2} \int_{\Sigma} a_{i j}\left(\nabla u_{i} \nabla u_{j}+8 \pi u_{j}\right) d V_{g} \\
& -\sum_{i=1}^{2} 4 \pi \log \int_{\Sigma} \exp \left(\sum_{j=1}^{2} a_{i j} u_{j}\right) d V_{g},
\end{aligned}
$$

and the Toda systems is

$$
-\Delta u_{i}=4 \pi\left(\frac{\exp \left(\sum_{j=1}^{2} a_{i j} u_{j}\right)}{\int_{\Sigma} \exp \left(\sum_{j=1}^{2} a_{i j} u_{j}\right)}-1\right), \text { for } 1 \leq i \leq 2,
$$

where $a_{11}=a_{22}=2$ and $a_{12}=a_{21}=-1$. The systems is equivalent to

$$
-\Delta u_{i}=4 \pi \sum_{j=1}^{2} a_{i j}\left(\frac{\exp \left(u_{j}\right)}{\int_{\Sigma} \exp \left(u_{j}\right)}-1\right), \text { for } 1 \leq i \leq 2,
$$

where $a_{11}=a_{22}=2$ and $a_{12}=a_{21}=-1$. 
Our main result is as follows:

Main Theorem. Let $\Sigma$ be a compact Riemann surface with area 1 . If the Gauss curvature $K$ of $\Sigma$ satisfies that

$$
\max _{p \in \Sigma} K(p)<2 \pi
$$

then $\Phi\left(u_{1}, u_{2}\right)$ has a minimizer.

We consider the sequence of minimizers $u^{\epsilon}=\left(u_{1}^{\epsilon}, u_{2}^{\epsilon}\right)$ of $\Phi_{(4 \pi-\epsilon, 4 \pi-\epsilon)}$ for small $\epsilon>0$. Then $u^{\epsilon}$ satisfies a Toda type systems. If $u^{\epsilon}$ converges to $u^{0}=\left(u_{1}^{0}, u_{2}^{0}\right)$ in $H_{2}:=H^{1,2}(\Sigma) \times H^{1,2}(\Sigma)$, then it is clear that $\Phi\left(u^{0}\right)=\inf _{u \in H_{2}} \Phi(u)$, i.e., $u^{0}$ is a minimizer of $\Phi$. If $u^{\epsilon}$ does not converge in $H_{2}$, in this case, we say that $u^{\epsilon}$ blows up. Then there are two cases happened according to Jost-Wang's result. For each case, we derive a delicate lower bound of $\Phi$ which is one of the main points in this paper. We apply capacity to calculate the lower bound, so that we need not know details in the neck. Such a trick has been used by the second author of this paper in [18], [19] to prove the existence of extremal functions for the classical Moser-Trudinger inequality on a compact manifold. Another main point of this paper is the delicate constructions of blowing up sequences $\phi^{\epsilon}$ in both cases, so that $\Phi\left(\phi^{\epsilon}\right)$ are strictly less than the lower bound derived before, and consequently we get a contradiction to the assumption that $u^{\epsilon}$ blows up, which proves our main theorem.

After submitting the present article we were informed of the existence of the paper [15], where a result similar to our one is established. As a matter of fact, Jost, Lin and Wang consider the more general equation

$$
-\Delta u_{i}=4 \pi \sum_{j=1}^{2} a_{i j}\left(\frac{h_{j} \exp \left(u_{j}\right)}{\int_{\Sigma} h_{j} \exp \left(u_{j}\right)}-1\right) \quad \text { for } 1 \leq i \leq 2
$$

where $a_{11}=a_{22}=2$ and $a_{12}=a_{21}=-1$, and $h_{1}, h_{2}$ are positive smooth functions on $\Sigma$. Our equation corresponds to the case where $h_{1} \equiv h_{2}$ is constant. Our approach to the proof is completely different from theirs, and it appears that the more general case considered by them could also be treated with our method. In addition, there are hopes that our techniques could be used to face other non-linear existence problems.

ACKNOWLEDGEMENTS. We thank the referee for his many helpful comments. 


\section{Review of known results}

For any $u=\left(u_{1}, u_{2}\right) \in H^{1,2}(\Sigma) \times H^{1,2}(\Sigma)$, we set

$$
\begin{aligned}
\Phi_{\epsilon}(u)= & \frac{1}{3} \int_{\Sigma}\left(\left|\nabla u_{1}\right|^{2}+\left|\nabla u_{2}\right|^{2}+\nabla u_{1} \nabla u_{2}+3(4 \pi-\epsilon) u_{1}+3(4 \pi-\epsilon) u_{2}\right) d V_{g} \\
& -(4 \pi-\epsilon) \log \int_{\Sigma} e^{u_{1}} d V_{g}-(4 \pi-\epsilon) \log \int_{\Sigma} e^{u_{2}} d V_{g} .
\end{aligned}
$$

It is not difficult to check that

$$
\Phi_{(4 \pi-\epsilon, 4 \pi-\epsilon)}(v)=\Phi_{\epsilon}(u),
$$

if we set $v_{1}=\frac{2 u_{1}+u_{2}}{3}$ and $v_{2}=\frac{u_{1}+2 u_{2}}{3}$.

By Jost-Wang's result ([16] Corollary 4.6), one sees that $\Phi_{\epsilon}$ has a minimizer $u^{\epsilon}$ of the functional $\Phi_{\epsilon}(u)$, i.e. we can find $u^{\epsilon} \in H^{1,2}(\Sigma) \times H^{1,2}(\Sigma)$ such that

$$
\Phi_{\epsilon}\left(u^{\epsilon}\right)=\inf \Phi_{\epsilon}(u) .
$$

Without loss of generality, we may assume that

$$
\int e^{u_{1}^{\epsilon}} d V_{g}=\int e^{u_{2}^{\epsilon}} d V_{g}=1
$$

Then we have the following equations:

$$
\left\{\begin{array}{l}
-\Delta u_{1}^{\epsilon}=(8 \pi-2 \epsilon) e^{u_{1}^{\epsilon}}-(4 \pi-\epsilon) e^{u_{2}^{\epsilon}}-(4 \pi-\epsilon) \\
-\Delta u_{2}^{\epsilon}=(8 \pi-2 \epsilon) e^{u_{2}^{\epsilon}}-(4 \pi-\epsilon) e^{u_{1}^{\epsilon}}-(4 \pi-\epsilon)
\end{array}\right.
$$

For $i=1,2$, let

$$
S_{i}=\left\{x \in \Sigma \text { : there is a sequence } y^{\epsilon} \rightarrow x \text { s.t. } u_{i}^{\epsilon}\left(y^{\epsilon}\right) \rightarrow+\infty\right\} .
$$

Jost-Wang [16] (section 5) proved that there will be two possibilities:

Case 1. $S_{1}=\left\{p_{1}\right\}$, and $S_{2}=\left\{p_{2}\right\}$, where $p_{1} p_{2}$ are two different points in $\Sigma$.

In this case, we set, for $i=1,2$,

$$
m_{i}^{\epsilon}=u_{i}^{\epsilon}\left(x_{i}^{\epsilon}\right)=\max u_{i}^{\epsilon}, \quad\left(r_{i}^{\epsilon}\right)^{2}=e^{-m_{i}^{\epsilon}}, \quad \bar{u}_{i}^{\epsilon}=\int_{\Sigma} u_{i}^{\epsilon} d V_{g} .
$$

Let $\left(\Omega_{i}, x=\left(x^{1}, x^{2}\right)\right)$ be an isothermal coordinate system around $p_{i}(i=1,2)$, and we assume the metric to be

$$
\left.g\right|_{\Omega_{i}}=e^{\varphi_{i}}\left(\left(d x^{1}\right)^{2}+\left(d x^{2}\right)^{2}\right)
$$

with $\varphi_{i}(0)=0, i=1,2$. 
We set, for $i=1,2, \Omega_{i}^{\epsilon}=\left\{x \in \mathbb{R}^{2}: x_{i}^{\epsilon}+r_{i}^{\epsilon} x \in \Omega_{i}\right\}$, which expands to the whole $\mathbb{R}^{2}$. In $\Omega_{1}^{\epsilon}$, we have the equations:

$$
\begin{aligned}
-\Delta_{0}\left(u_{1}^{\epsilon}\left(x_{1}^{\epsilon}+r_{1}^{\epsilon} x\right)-m_{1}^{\epsilon}\right)= & e^{-\varphi_{1}\left(x_{1}^{\epsilon}+r_{1}^{\epsilon} x\right)}\left((8 \pi-2 \epsilon) e^{u_{1}^{\epsilon}\left(x_{1}^{\epsilon}+r_{1}^{\epsilon} x\right)-m_{1}^{\epsilon}}\right. \\
& \left.-\left(r_{1}^{\epsilon}\right)^{2}(4 \pi-\epsilon) e^{u_{2}^{\epsilon}\left(x_{1}^{\epsilon}+r_{1}^{\epsilon} x\right)}-\left(r_{1}^{\epsilon}\right)^{2}(4 \pi-\epsilon)\right),
\end{aligned}
$$

where $-\Delta_{0}=\frac{\partial^{2}}{\partial^{2} x^{1}}+\frac{\partial^{2}}{\partial^{2} x^{2}}$. Since $u_{2}^{\epsilon}$ are bounded from above in $\Omega_{1}^{\epsilon}$, it follows from the Harnack inequality and the elliptic estimates that $u_{1}^{\epsilon}$ converges in $C_{\mathrm{loc}}^{k}\left(\mathbb{R}^{2}\right)$ for any $k$ to the function $w$ which satisfies the equation

$$
\left\{\begin{array}{l}
-\Delta w=8 \pi e^{w}, \quad \forall x \in \mathbb{R}^{2} \\
w(x) \leq w(0)=0, \quad \text { and } \int_{\mathbb{R}^{2}} e^{w} d x \leq 1 .
\end{array}\right.
$$

Hence, by the result in [7], we know that

$$
w=-2 \log \left(1+\pi|x|^{2}\right) .
$$

In the same way, $u_{2}^{\epsilon}\left(x_{2}^{\epsilon}+r_{2}^{\epsilon} x\right)-m_{2}^{\epsilon}$ converges to $w$.

Setting $\bar{u}_{i}^{\epsilon}=\int_{\Sigma} u_{i}^{\epsilon} d V_{g}$, we have the following proposition (see Lemma 5.6, and the proof of Theorem 3.1 in [16]).

Proposition 2.1. We have $\bar{u}_{j}^{\epsilon} \rightarrow-\infty$ for $j=1,2$. Furthermore, for any $q \in$ $(1,2)$, we have

$$
u_{j}^{\epsilon}-\bar{u}_{j}^{\epsilon} \text { converges to } G_{j} \text { in } H^{1, q}(\Sigma),
$$

where $G_{1}$ and $G_{2}$ satisfy

$$
\left\{\begin{aligned}
-\Delta G_{1} & =8 \pi \delta_{p_{1}}-4 \pi \delta_{p_{2}}-4 \pi \\
-\Delta G_{2} & =8 \pi \delta_{p_{2}}-4 \pi \delta_{p_{1}}-4 \pi, \\
\int_{\Sigma} G_{j} d V_{g} & =0, \quad \text { for } j=1,2
\end{aligned}\right.
$$

where $\delta_{y}$ is the Dirac distribution. Moreover,

$$
u_{j}^{\epsilon}-\bar{u}_{j}^{\epsilon} \text { converges to } G_{j} \text { in } C_{\mathrm{loc}}^{2}\left(\Sigma \backslash\left\{p_{1}, p_{2}\right\}\right) .
$$

Remark 2.2. It is easy to see that, in $\Omega_{1}$,

$$
G_{1}=-4 \log r+A_{1}\left(p_{1}\right)+f_{1}, \text { and } G_{2}=2 \log r+A_{2}\left(p_{1}\right)+g_{1}
$$

where $r^{2}=x_{1}^{2}+x_{2}^{2}, A_{i}\left(p_{1}\right)(i=1,2)$ are constants, and $f_{1}, g_{1}$ are smooth functions which are zero at 0 . Similarly, in $\Omega_{2}$, we can write

$$
G_{1}=2 \log r+A_{1}\left(p_{2}\right)+f_{2}, \quad \text { and } G_{2}=-4 \log r+A_{2}\left(p_{2}\right)+g_{2}
$$

where $A_{i}\left(p_{1}\right)(i=1,2)$ are constants, and $f_{2}, g_{2}$ are smooth functions which are zero at 0 . 
Case 2. $S_{1}=\{p\}$, and $S_{2}=\emptyset$.

In this case, $u_{2}^{\epsilon}$ are bounded from above. Let $(\Omega ; x)$ be an isothermal coordinate system around $p$, similar to the Case 1 , we have

$$
u_{1}^{\epsilon}\left(x_{1}^{\epsilon}+r_{1}^{\epsilon} x\right)-m_{1}^{\epsilon} \rightarrow-2 \log \left(1+\pi|x|^{2}\right) .
$$

We also have the following proposition (c.f. [16]):

Proposition 2.3. Let $\bar{u}_{1}^{\epsilon}$ be the average of $u_{1}^{\epsilon}$. We have $\bar{u}_{1}^{\epsilon} \rightarrow-\infty$. Furthermore, for any $q \in(1,2)$, we have

$$
u_{1}^{\epsilon}-\bar{u}_{1}^{\epsilon} \text { converges to } G_{1} \text { in } H^{1, q}(\Sigma),
$$

and

$$
u_{2}^{\epsilon} \text { converges to } G_{2} \text { in } H^{1, q}(\Sigma),
$$

where $G_{1}$ and $G_{2}$ satisfy

$$
\left\{\begin{aligned}
-\Delta G_{1} & =8 \pi \delta_{p}-4 \pi e^{G_{2}}-4 \pi \\
-\Delta G_{2} & =8 \pi e^{G_{2}}-4 \pi \delta_{p}-4 \pi \\
\int_{\Sigma} G_{1} d V_{g} & =0, \quad \int_{\Sigma} e^{G_{2}} d V_{g}=1, \quad \sup _{x \in \Sigma} G_{2}<+\infty
\end{aligned}\right.
$$

where $\delta_{y}$ is the Dirac distribution. Moreover,

$$
u_{1}^{\epsilon}-\bar{u}_{1}^{\epsilon} \text { converges to } G_{1} \text {, and } u_{2}^{\epsilon} \text { converges to } G_{2} \text { in } C_{\text {loc }}^{2}(\Sigma \backslash\{p\}) \text {. }
$$

Since $G_{2}$ is bounded from above, we can deduce from the equation (2.3) that $G_{2}=$ $2 \log r+h$ in $\Omega$, where $h \in H_{\mathrm{loc}}^{2, q}(\Omega)$ for any $q>0$. Then $e^{G_{2}}=r^{2} e^{h} \in C_{\mathrm{loc}}^{1}(\Omega)$, and then $\Delta_{0} h \in C_{\text {loc }}^{1}(\Omega)$. Therefore, by the standard elliptic estimates, $G_{2}-2 \log r$ is smooth in $\Omega$. So, we can write

$$
G_{1}=-4 \log r+A_{1}(p)+f, \quad \text { and } \quad G_{2}=2 \log r+A_{2}(p)+g
$$

where $r^{2}=x_{1}^{2}+x_{2}^{2}, A_{i}(p)(i=1,2)$ are constants and $f, g$ are smooth functions which are zero at 0 .

\section{The lower bound for Case 1}

We assume that $\Omega_{1} \cap \Omega_{2}=\emptyset$, and $B_{r}\left(p_{1}\right) \subset \Omega_{1}$. We set $v_{2}^{\epsilon}=\frac{1}{3}\left(2 u_{2}^{\epsilon}+u_{1}^{\epsilon}\right)-$ $\frac{1}{3}\left(2 \bar{u}_{2}^{\epsilon}+\bar{u}_{1}^{\epsilon}\right)$. Then, in $B_{r}\left(p_{1}\right)$, we have

$$
\left\{\begin{array}{l}
-\Delta v_{2}^{\epsilon}=(4 \pi-\epsilon) e^{u_{2}^{\epsilon}}-(4 \pi-\epsilon) \in L^{\infty}\left(B_{r}\left(p_{1}\right)\right) \\
\left.v_{2}^{\epsilon}\right|_{\partial B_{r}\left(p_{1}\right)} \rightarrow \frac{1}{3}\left(2 G_{2}+G_{1}\right) .
\end{array}\right.
$$

So $\left\|v_{2}^{\epsilon}\right\|_{C^{1}} \leq C$, where $C$ is a constant depending only on $r$. 
By a direct calculation one gets

$$
\begin{aligned}
\frac{1}{3} \int_{B_{\delta}\left(x_{1}^{\epsilon}\right)}\left(\left|\nabla u_{1}\right|^{2}+\left|\nabla u_{2}\right|^{2}+\nabla u_{1} \nabla u_{2}\right) d V_{g} & =\frac{1}{4} \int_{B_{\delta}\left(x_{k}\right)}\left(\left|\nabla u_{1}^{\epsilon}\right|^{2}+3\left|\nabla v_{2}^{\epsilon}\right|^{2}\right) d V_{g} \\
& =\frac{1}{4} \int_{B_{\delta}\left(x_{1}^{\epsilon}\right)}\left|\nabla u_{1}^{\epsilon}\right|^{2} d V_{g}+O\left(\delta^{2}\right) .
\end{aligned}
$$

Recall that $u_{1}\left(x_{1}^{\epsilon}+r_{1}^{\epsilon} x\right)-m_{1}^{\epsilon} \rightarrow w$ in $C^{k}\left(B_{L}(0)\right)$, for any $k$, we have

$$
\begin{aligned}
\frac{1}{4} \int_{B_{\delta}\left(x_{1}^{\epsilon}\right)}\left|\nabla u_{1}^{\epsilon}\right|^{2} d V_{g}= & \frac{1}{4} \int_{B_{L}}|\nabla w|^{2} d x \\
& +\frac{1}{4} \int_{B_{\delta}\left(x_{1}^{\epsilon}\right) \backslash B_{L r_{1}^{\epsilon}\left(x_{1}^{\epsilon}\right)}}\left|\nabla u_{1}^{\epsilon}\right|^{2} d V_{g}+o(1)+O\left(\delta^{2}\right) .
\end{aligned}
$$

Let

$$
a_{1}^{\epsilon}=\inf _{\partial B_{L r_{1}^{\epsilon}}\left(x_{1}^{\epsilon}\right)} u_{1}^{\epsilon}, \quad b_{1}^{\epsilon}=\sup _{\partial B_{\delta}\left(x_{1}^{\epsilon}\right)} u_{1}^{\epsilon} .
$$

We set $a_{1}^{\epsilon}-b_{1}^{\epsilon}=m_{1}^{\epsilon}-\bar{u}_{1}^{\epsilon}+d_{1}^{\epsilon}$. It is clear that, for fixed $L$ and $\delta$,

$$
d_{1}^{\epsilon} \rightarrow w(L)-\sup _{\partial B_{\delta}\left(p_{1}\right)} G_{1} \text { as } \epsilon \rightarrow 0 .
$$

Let $f_{1}^{\epsilon}=\max \left\{\min \left\{u_{1}^{\epsilon}, a_{1}^{\epsilon}\right\}, b_{1}^{\epsilon}\right\}$. We get

$$
\begin{aligned}
\int_{B_{\delta}\left(x_{1}^{\epsilon}\right) \backslash B_{L r_{1}^{\epsilon}}\left(x_{1}^{\epsilon}\right)}\left|\nabla u_{1}^{\epsilon}\right|^{2} d V_{g} & \geq \int_{B_{\delta}\left(x_{1}^{\epsilon}\right) \backslash B_{L r_{1}^{\epsilon}}\left(x_{1}^{\epsilon}\right)}\left|\nabla f_{1}^{\epsilon}\right|^{2} d V_{g} \\
& =\int_{B_{\delta}\left(x_{1}^{\epsilon}\right) \backslash B_{L r_{1}^{\epsilon}}\left(x_{1}^{\epsilon}\right)}\left|\nabla_{0} f_{1}^{\epsilon}\right|^{2} d x \\
& \geq \inf _{\left.\Psi\right|_{L r_{1}^{\epsilon}}(0)=a_{1}^{\epsilon},\left.\Psi\right|_{\partial B_{\delta}(0)}=b_{1}^{\epsilon}} \int_{B_{\delta}(0) \backslash B_{L r_{1}^{\epsilon}}(0)}\left|\nabla_{0} \Psi\right|^{2} d x .
\end{aligned}
$$

Here, $\left|\nabla_{0} g\right|^{2}=\left|\frac{\partial g}{\partial x_{1}}\right|^{2}+\left|\frac{\partial g}{\partial x_{2}}\right|^{2}$. It is well-known that

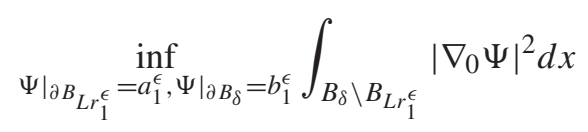


is uniquely attained by the function $\phi$ which satisfies the equation

$$
\left\{\begin{array}{l}
-\Delta_{0} \phi=0 \\
\left.\phi\right|_{\partial B_{L r_{1}^{\epsilon}}}=a_{1}^{\epsilon},\left.\phi\right|_{\partial B_{\delta}}=b_{1}^{\epsilon} .
\end{array}\right.
$$

Hence,

$$
\phi=\frac{a_{1}^{\epsilon}-b_{1}^{\epsilon}}{-\log L r_{1}^{\epsilon}+\log \delta} \log r+\frac{a_{1}^{\epsilon} \log \delta-b_{1}^{\epsilon} \log L r_{k}}{-\log L r_{1}^{\epsilon}+\log \delta},
$$

and then

$$
\int_{B_{\delta}(0) \backslash B_{L r_{1}^{\epsilon}}(0)}\left|\nabla_{0} \phi\right|^{2} d x=\frac{4 \pi\left(a_{1}^{\epsilon}-b_{1}^{\epsilon}\right)^{2}}{-\log \left(L r_{1}^{\epsilon}\right)^{2}+\log \delta^{2}} .
$$

Therefore, we have

$$
\int_{B_{\delta}\left(x_{1}^{\epsilon}\right) \backslash B_{L r_{1}^{\epsilon}\left(x_{1}^{\epsilon}\right)}}\left|\nabla u_{1}^{\epsilon}\right|^{2} d V_{g} \geq \frac{4 \pi\left(m_{1}^{\epsilon}-\bar{u}_{1}^{\epsilon}+d_{1}^{\epsilon}\right)^{2}}{-\log L^{2}-\log \left(r_{1}^{\epsilon}\right)^{2}+\log \delta^{2}} .
$$

Recalling that $-\log \left(r_{1}^{\epsilon}\right)^{2}=m_{1}^{\epsilon}$, we get

$$
\begin{aligned}
\int_{B_{\delta}\left(x_{1}^{\epsilon}\right) \backslash B_{L r_{1}^{\epsilon}\left(x_{1}^{\epsilon}\right)}\left|\nabla u_{1}^{\epsilon}\right|^{2} d V_{g} \geq} & 4 \pi \frac{\left(m_{1}^{\epsilon}-\bar{u}_{1}^{\epsilon}+d_{1}^{\epsilon}\right)^{2}}{m_{1}^{\epsilon}}\left(1-\frac{\log L^{2}-\log \delta^{2}}{m_{1}^{\epsilon}}\right)^{-1} \\
\geq & 4 \pi \frac{\left(m_{1}^{\epsilon}-\bar{u}_{1}^{\epsilon}+d_{1}^{\epsilon}\right)^{2}}{m_{1}^{\epsilon}}\left(1+\frac{\log L^{2}-\log \delta^{2}}{m_{1}^{\epsilon}}+\frac{A}{\left(m_{1}^{\epsilon}\right)^{2}}\right) \\
\geq & 4 \pi \frac{\left(m_{1}^{\epsilon}-\bar{u}_{1}^{\epsilon}\right)^{2}}{m_{1}^{\epsilon}}+8 \pi d_{1}^{\epsilon}\left(1-\frac{\bar{u}_{1}^{\epsilon}}{m_{1}^{\epsilon}}\right) \\
& +4 \pi\left(1-\frac{\bar{u}_{1}^{\epsilon}}{m_{1}^{\epsilon}}\right)^{2}\left(\log L^{2}-\log \delta^{2}\right)+\frac{A^{\prime} \bar{u}_{1}^{\epsilon}}{\left(m_{1}^{\epsilon}\right)^{2}}
\end{aligned}
$$

where $A$ and $A^{\prime}$ are constants which depend only on $\delta$ and $L$.

Then we have

$$
\begin{aligned}
& \frac{1}{3} \int_{B_{\delta}\left(x_{1}^{\epsilon}\right)}\left(\left|\nabla u_{1}\right|^{2}+\left|\nabla u_{2}\right|^{2}+\nabla u_{1} \nabla u_{2}\right) d V_{g} \geq \frac{1}{4} \int_{B_{L}}|\nabla w|^{2} d x \\
& +\pi \frac{\left(m_{1}^{\epsilon}-\bar{u}_{1}^{\epsilon}\right)^{2}}{m_{1}^{\epsilon}}+2 \pi d_{1}^{\epsilon}\left(1-\frac{\bar{u}_{1}^{\epsilon}}{m_{1}^{\epsilon}}\right)+\pi\left(1-\frac{\bar{u}_{1}^{\epsilon}}{m_{1}^{\epsilon}}\right)^{2}\left(\log L^{2}-\log \delta^{2}\right) \\
& \quad+\frac{A^{\prime}(\delta, L) \bar{u}_{1}^{\epsilon}}{\left(m_{1}^{\epsilon}\right)^{2}}+o(1)+O\left(\delta^{2}\right) .
\end{aligned}
$$


Similarly, we have

$$
\begin{aligned}
& \frac{1}{3} \int_{B_{\delta}\left(x_{2}^{\epsilon}\right)}\left(\left|\nabla u_{1}\right|^{2}+\left|\nabla u_{2}\right|^{2}+\nabla u_{1} \nabla u_{2}\right) d V_{g} \\
& \geq \frac{1}{4} \int_{B_{L}}|\nabla w|^{2} d x+\pi \frac{\left(m_{2}^{\epsilon}-\bar{u}_{2}^{\epsilon}\right)^{2}}{m_{2}^{\epsilon}}+2 \pi d_{1}^{\epsilon}\left(1-\frac{\bar{u}_{2}^{\epsilon}}{m_{2}^{\epsilon}}\right) \\
& \quad+\pi\left(1-\frac{\bar{u}_{2}^{\epsilon}}{m_{1}^{\epsilon}}\right)^{2}\left(\log L^{2}-\log \delta^{2}\right)+\frac{A^{\prime} \bar{u}_{2}^{\epsilon}}{\left(m_{2}^{\epsilon}\right)^{2}}+o(1)+O\left(\delta^{2}\right) .
\end{aligned}
$$

It follows that

$$
\begin{aligned}
& \frac{1}{3} \int_{B_{\delta}\left(x_{1}^{\epsilon}\right) \cup B_{\delta}\left(x_{2}^{\epsilon}\right)}\left(\left|\nabla u_{1}\right|^{2}+\left|\nabla u_{2}\right|^{2}+\nabla u_{1} \nabla u_{2}\right) d V_{g}+(4 \pi-\epsilon) \bar{u}_{1}^{\epsilon}+(4 \pi-\epsilon) \bar{u}_{2}^{\epsilon} \\
& \geq \frac{1}{3} \int_{B_{\delta}\left(x_{1}^{\epsilon}\right) \cup B_{\delta}\left(x_{2}^{\epsilon}\right)}\left(\left|\nabla u_{1}\right|^{2}+\left|\nabla u_{2}\right|^{2}+\nabla u_{1} \nabla u_{2}\right) d V_{g}+4 \pi \bar{u}_{1}^{\epsilon}+4 \pi \bar{u}_{2}^{\epsilon} \\
& \geq \frac{1}{2} \int_{B_{L}}|\nabla w|^{2} d x \\
& \quad+\sum_{i=1,2}\left(\pi \frac{\left(m_{i}^{\epsilon}+\bar{u}_{i}^{\epsilon}\right)^{2}}{m_{i}^{\epsilon}}+2 \pi d_{i}^{\epsilon}\left(1-\frac{\bar{u}_{i}^{\epsilon}}{m_{i}^{\epsilon}}\right)+\pi\left(1-\frac{\bar{u}_{2}^{\epsilon}}{m_{1}^{\epsilon}}\right)^{2}\left(\log L^{2}-\log \delta^{2}\right)\right) \\
& \quad+\sum_{i=1,2} \frac{A^{\prime} \bar{u}_{i}^{\epsilon}}{\left(m_{i}^{\epsilon}\right)^{2}}+o(1)+O\left(\delta^{2}\right) \\
& \geq \frac{1}{2} \int_{B_{L}}|\nabla w|^{2} d x \\
& \quad+\sum_{i=1,2}\left(\pi m_{i}^{\epsilon}\left(1+\frac{\bar{u}_{i}^{\epsilon}}{m_{i}^{\epsilon}}\right)^{2}+2 \pi d_{i}^{\epsilon}\left(1-\frac{\bar{u}_{i}^{\epsilon}}{m_{i}^{\epsilon}}\right)+\pi\left(1-\frac{\bar{u}_{2}^{\epsilon}}{m_{1}^{\epsilon}}\right)^{2}\left(\log L^{2}-\log \delta^{2}\right)\right) \\
& \quad+\sum_{i=1,2} \frac{A^{\prime} \bar{u}_{i}^{\epsilon}}{\left(m_{i}^{\epsilon}\right)^{2}}+o(1)+O\left(\delta^{2}\right) .
\end{aligned}
$$

We now set $s_{i}^{\epsilon}=1+\frac{\bar{u}_{i}^{\epsilon}}{m_{i}^{\epsilon}}$. Then, for fixed $L, \delta$, we have

$$
\begin{aligned}
\frac{1}{3} \int_{\Sigma}\left(\left|\nabla u_{1}\right|^{2}+\left|\nabla u_{2}\right|^{2}\right. & \left.+\nabla u_{1} \nabla u_{2}\right) d V_{g}+(4 \pi-\epsilon) \bar{u}_{1}^{\epsilon}+(4 \pi-\epsilon) \bar{u}_{2}^{\epsilon} \\
& \geq \sum_{i} m_{i}^{\epsilon}\left(s_{i}^{\epsilon}+O\left(\frac{1}{m_{i}^{\epsilon}}\right)\right)^{2}+C
\end{aligned}
$$

Since $\Phi_{\epsilon}\left(u_{\epsilon}\right) \leq C$, we see that

$$
\left|s_{i}^{\epsilon}\right|=O\left(\frac{1}{m_{i}^{\epsilon}}\right) .
$$


Hence for both $i=1,2, s_{i}^{\epsilon} \rightarrow 0$ as $\epsilon \rightarrow 0$. So,

$$
\begin{aligned}
& \frac{1}{3} \int_{B_{\delta}\left(x_{1}^{\epsilon}\right) \cup B_{\delta}\left(x_{2}^{\epsilon}\right)}\left(\left|\nabla u_{1}\right|^{2}+\left|\nabla u_{2}\right|^{2}+\nabla u_{1} \nabla u_{2}\right) d V_{g}+(4 \pi-\epsilon) \bar{u}_{1}^{\epsilon}+(4 \pi-\epsilon) \bar{u}_{2}^{\epsilon} \\
\geq & \frac{1}{2} \int_{B_{L}}|\nabla w|^{2} d x+4 \pi d_{1}^{\epsilon}+4 \pi d_{2}^{\epsilon}+8 \pi\left(\log L^{2}-\log \delta^{2}\right)+o(1)+O\left(\delta^{2}\right) \\
= & \frac{1}{2} \int_{B_{L}}|\nabla w|^{2} d x+8 \pi w(L)+8 \pi\left(\log L^{2}-\log \delta^{2}\right) \\
& -4 \pi \sup _{\partial B_{\delta}\left(p_{1}\right)} G_{1}-4 \pi \sup _{\partial B_{\delta}\left(p_{2}\right)} G_{2}+o(1)+O\left(\delta^{2}\right) .
\end{aligned}
$$

By a direct calculation, we obtain

$$
\int_{B_{L}}|\nabla w|^{2} d x=16 \pi \log \left(1+\pi L^{2}\right)-\frac{16 \pi^{2} L^{2}}{1+\pi L^{2}} .
$$

Moreover, by (2.1) and (2.2), we have

$$
\begin{aligned}
& \frac{1}{3} \int_{B_{\delta}^{c}\left(x_{1}^{\epsilon}\right) \cap B_{\delta}^{c}\left(x_{2}^{\epsilon}\right)}\left(\left|\nabla u_{1}\right|^{2}+\left|\nabla u_{2}\right|^{2}+\nabla u_{1} \nabla u_{2}\right) d V_{g} \\
= & \frac{1}{3} \int_{B_{\delta}^{c}\left(x_{1}^{\epsilon}\right) \cap B_{\delta}^{c}\left(x_{2}^{\epsilon}\right)}\left(\left|\nabla G_{1}\right|^{2}+\left|\nabla G_{2}\right|^{2}+\nabla G_{1} \nabla G_{2}\right) d V_{g}+o(1) \\
= & -\frac{1}{3} \sum_{i=1,2} \int_{\partial B_{\delta}\left(p_{i}\right)}\left(G_{1} \frac{\partial G_{1}}{\partial n}+G_{2} \frac{\partial G_{2}}{\partial n}+\frac{G_{1} \frac{\partial G_{2}}{\partial n}+G_{2} \frac{\partial G_{1}}{\partial n}}{2}\right) d S_{g}+o(1) \\
& +\int_{B_{\delta}\left(p_{1}\right)+B_{\delta}\left(p_{2}\right)} 2 \pi\left(G_{1}+G_{2}\right) d V_{g}+o(1) \\
= & -\left.\frac{1}{3} \sum_{i=1,2} \int_{0}^{2 \pi}\left(G_{1} \frac{\partial G_{1}}{\partial r}+G_{2} \frac{\partial G_{2}}{\partial r}+\frac{G_{1} \frac{\partial G_{2}}{\partial r}+G_{2} \frac{\partial G_{1}}{\partial r}}{2}\right) r d \theta\right|_{r=\delta}+o(1) \\
& +\int_{B_{\delta}\left(p_{1}\right)+B_{\delta}\left(p_{2}\right)} 2 \pi\left(G_{1}+G_{2}\right) d V_{g}+o(1) \\
= & -16 \pi \log \delta-2 \pi A_{1}\left(p_{1}\right)-2 \pi A_{2}\left(p_{2}\right)+o(1)+O(\delta \log \delta) .
\end{aligned}
$$

In the end, (3.1), (3.2) and (3.3) imply that

$$
\inf \Phi_{0}(u) \geq-8 \pi \log \pi-8 \pi-2 \pi\left(A_{1}\left(p_{1}\right)+A_{2}\left(p_{2}\right)\right) .
$$




\section{Lower bound for Case 2}

In this case we set $v_{2}^{\epsilon}=\frac{1}{3}\left(2 u_{2}^{\epsilon}+u_{1}^{\epsilon}\right)-\frac{1}{3}\left(2 \bar{u}_{2}^{\epsilon}+\bar{u}_{1}^{\epsilon}\right)$. Then we have

$$
\left\{\begin{array}{l}
-\Delta v_{2}^{\epsilon}=(4 \pi-\epsilon) e^{u_{2}^{\epsilon}}-(4 \pi-\epsilon) \\
\int v_{2}^{\epsilon}=0
\end{array}\right.
$$

By the standard elliptic estimates, $\left\|v_{2}\right\|_{C^{1}(M)}<C$.

Similarly to Case 1 , we have

$$
\frac{1}{3} \int_{B_{\delta}\left(x_{1}^{\epsilon}\right)}\left(\left|\nabla u_{1}\right|^{2}+\left|\nabla u_{2}\right|^{2}+\nabla u_{1} \nabla u_{2}\right) d V_{g}=\frac{1}{4} \int_{B_{\delta}\left(x_{1}^{\epsilon}\right)}\left|\nabla u_{1}^{\epsilon}\right|^{2} d V_{g}+O\left(\delta^{2}\right),
$$

and

$$
\begin{aligned}
& \frac{1}{4} \int_{B_{\delta}\left(x_{1}^{\epsilon}\right)}\left|\nabla u_{1}\right|^{2} d V_{g}+(4 \pi-\epsilon) \bar{u}_{1}^{\epsilon} \geq \frac{1}{4} \int_{B_{L}}|\nabla w|^{2} d x \\
& \quad+\left(\pi \frac{\left(m_{1}^{\epsilon}+\bar{u}_{1}^{\epsilon}\right)^{2}}{m_{1}^{\epsilon}}+2 \pi d_{1}^{\epsilon}\left(1-\frac{\bar{u}_{1}^{\epsilon}}{m_{1}^{\epsilon}}\right)+\pi\left(1-\frac{\bar{u}_{1}^{\epsilon}}{m_{1}^{\epsilon}}\right)^{2}\left(\log L^{2}-\log \delta^{2}\right)\right) \\
& +\frac{A^{\prime} \bar{u}_{1}^{\epsilon}}{\left(m_{1}^{\epsilon}\right)^{2}}+o(1)+O\left(\delta^{2}\right) .
\end{aligned}
$$

By an argument similar to that used in Case 1, we can show that $\frac{\bar{u}_{1}^{\epsilon}}{m_{1}^{\epsilon}} \rightarrow-1$, hence

$$
\begin{aligned}
& \frac{1}{3} \int_{B_{\delta}\left(x_{1}^{\epsilon}\right)}\left(\left|\nabla u_{1}\right|^{2}+\left|\nabla u_{2}\right|^{2}+\nabla u_{1} \nabla u_{2}\right) d V_{g}+(4 \pi-\epsilon) \bar{u}_{1}^{\epsilon} \\
& \geq \frac{1}{4} \int_{B_{L}}|\nabla w|^{2} d x+4 \pi w(L) \\
& \quad+4 \pi\left(\log L^{2}-\log \delta^{2}\right)-4 \pi \sup _{\partial B_{\delta}\left(p_{1}\right)} G_{1}+o(1)+O\left(\delta^{2}\right) .
\end{aligned}
$$

Set

$$
G_{1}=-4 \log r+A_{1}(p)+o(x), \quad G_{2}=2 \log r+A_{2}(p)+o(x) .
$$


Applying (2.4), we get

$$
\begin{aligned}
\frac{1}{3} & \int_{B_{\delta}^{c}\left(x_{1}^{\epsilon}\right)}\left(\left|\nabla u_{1}\right|^{2}+\left|\nabla u_{2}\right|^{2}+\nabla u_{1} \nabla u_{2}\right) d V_{g} \\
= & \frac{1}{3} \int_{B_{\delta}^{c}\left(x_{1}^{\epsilon}\right)}\left(\left|\nabla G_{1}\right|^{2}+\left|\nabla G_{2}\right|^{2}+\frac{\nabla G_{1} \nabla G_{2}+\nabla G_{2} \nabla G_{1}}{2}\right) d V_{g}+o(1) \\
= & \int_{\partial B_{\delta}(p)}\left(G_{1} \frac{\partial G_{1}}{\partial n}+G_{2} \frac{\partial G_{2}}{\partial n}+\frac{G_{1} \frac{\partial G_{2}}{\partial n}+G_{2} \frac{\partial G_{1}}{\partial n}}{2}\right) \\
& +2 \pi \int_{B_{\delta}(p)}\left(G_{1}+G_{2}\right) d V_{g}-2 \pi \int_{\Sigma} G_{2} d V_{g}+o(1)+O(\delta \log \delta) \\
= & -8 \pi \log \delta-2 \pi A_{1}\left(p_{1}\right)-2 \pi \int_{\Sigma} G_{2} d V_{g}+o(1)+O(\delta \log \delta) .
\end{aligned}
$$

In the end, we obtain

$$
\inf \Phi_{0}(u) \geq-4 \pi \log \pi-2 \pi A_{1}(p)+2 \pi \int G_{2} d V_{g} .
$$

\section{Test functions for Case 1}

In this section we will construct a function $\phi=\left(\phi_{1}, \phi_{2}\right) \in H^{1,2}(M) \times H^{1,2}(M)$, such that

$$
\Phi_{0}(\phi)<-8 \pi \log \pi-8 \pi-2 \pi\left(A_{2}\left(p_{1}\right)+A_{1}\left(p_{2}\right)\right),
$$

whenever (1.4) holds. So, under assumption (1.4), Case 1 will not occur.

Let $\left(\Omega_{i} ;(x, y)\right)$ be an isothermal coordinate system around $p_{i}(i=1,2)$. We set

$$
r(x, y)=\sqrt{x^{2}+y^{2}}, \text { and } B_{\delta}=\left\{(x, y): x^{2}+y^{2}<\delta^{2}\right\} .
$$

We assume that near $p_{i}(i=1,2)$, for each $k=1,2$,

$$
\begin{aligned}
G_{k}= & a_{k}\left(p_{i}\right) \log r+A_{k}\left(p_{i}\right)+\lambda_{k}\left(p_{i}\right) x+\mu_{k}\left(p_{i}\right) y \\
& +\alpha_{k}\left(p_{i}\right) x^{2}+\beta_{k}\left(p_{i}\right) y^{2}+\gamma_{k}\left(p_{i}\right) x y+h(x, y)+O\left(r^{4}\right) .
\end{aligned}
$$

We have $a_{1}\left(p_{1}\right)=a_{2}\left(p_{2}\right)=-4$, and $a_{1}\left(p_{2}\right)=a_{2}\left(p_{1}\right)=2$. Moreover, we assume that

$$
\left.g\right|_{\Omega_{i}}=e^{\varphi_{i}}\left(d x^{2}+d y^{2}\right),
$$

and

$$
\varphi_{i}=b_{1}\left(p_{i}\right) x+b_{2}\left(p_{i}\right) y+c_{1}\left(p_{i}\right) x^{2}+c_{2}\left(p_{i}\right) y^{2}+c_{12}\left(p_{i}\right) x y+O\left(r^{3}\right) .
$$

It is well known that

$$
\begin{aligned}
K\left(p_{i}\right) & =-\left(c_{1}\left(p_{i}\right)+c_{2}\left(p_{i}\right)\right), \\
|\nabla u|^{2} d V_{g} & =|\nabla u|^{2} d x d y,
\end{aligned}
$$


and

$$
\frac{\partial u}{\partial n} d S_{g}=\frac{\partial u}{\partial r} r d \theta, \quad\left(S=\partial B_{r}\right) .
$$

For $\alpha_{k}$ and $\beta_{k}$, we have the following lemma:

Lemma 5.1. For any $k$, we have

$$
\alpha_{k}\left(p_{i}\right)+\beta_{k}\left(p_{i}\right)=2 \pi .
$$

Proof. Near $p_{i}$ we have

$$
2 \alpha_{k}\left(p_{i}\right)+2 \beta_{k}\left(p_{i}\right)+O(r)=\Delta_{0} G_{k}(x, y)=e^{-\varphi_{i}} 4 \pi .
$$

We choose

$$
\phi_{1}= \begin{cases}w\left(\frac{x}{\epsilon}\right)+\lambda_{1}\left(p_{1}\right) r \cos \theta+\mu_{1}\left(p_{1}\right) r \sin \theta & (x, y) \in B_{L \epsilon}\left(p_{1}\right) \\ G_{1}-\eta_{1} H_{1}^{p_{1}}+4 \log L \epsilon-2 \log \left(1+\pi L^{2}\right)-A_{1}\left(p_{1}\right) & (x, y) \in B_{2 L \epsilon} \backslash B_{L \epsilon}\left(p_{1}\right) \\ G_{1}-\eta_{2} H_{1}^{p_{2}}+4 \log L \epsilon-2 \log \left(1+\pi L^{2}\right)-A_{1}\left(p_{1}\right) & (x, y) \in B_{2 L \epsilon} \backslash B_{L \epsilon}\left(p_{2}\right) \\ -\frac{x\left(\frac{x}{\epsilon}\right)+2 \log \left(1+\pi L^{2}\right)}{2}+\lambda_{1}\left(p_{2}\right) r \cos \theta+\mu_{1}\left(p_{2}\right) r \sin \theta & (x, y) \in B_{L \epsilon}\left(p_{2}\right) \\ +6 \log L \epsilon-2 \log \left(1+\pi L^{2}\right)+A_{1}\left(p_{2}\right)-A_{1}\left(p_{1}\right) & \text { otherwise, }\end{cases}
$$

and

$$
\phi_{2}= \begin{cases}w\left(\frac{x}{\epsilon}\right)+\lambda_{2}\left(p_{2}\right) r \cos \theta+\mu_{2}\left(p_{2}\right) r \sin \theta & (x, y) \in B_{L \epsilon}\left(p_{2}\right) \\ G_{2}-\eta_{2} H_{2}^{p_{2}}+4 \log L \epsilon-2 \log \left(1+\pi L^{2}\right)-A_{2}\left(p_{2}\right) & (x, y) \in B_{2 L \epsilon} \backslash B_{L \epsilon}\left(p_{2}\right) \\ G_{2}-\eta_{1} H_{2}^{p_{1}}+4 \log L \epsilon-2 \log \left(1+\pi L^{2}\right)-A_{2}\left(p_{2}\right) & (x, y) \in B_{2 L \epsilon} \backslash B_{L \epsilon}\left(p_{1}\right) \\ -\frac{\omega\left(\frac{x}{\epsilon}\right)+2 \log \left(1+\pi L^{2}\right)}{2}+\lambda_{2}\left(p_{1}\right) r \cos \theta+\mu_{2}\left(p_{1}\right) r \sin \theta \\ +6 \log L \epsilon-2 \log \left(1+\pi L^{2}\right)+A_{2}\left(p_{1}\right)-A_{2}\left(p_{2}\right) & (x, y) \in B_{L \epsilon}\left(p_{1}\right) \\ G_{2}+4 \log L \epsilon-2 \log \left(1+\pi L^{2}\right)-A_{2}\left(p_{2}\right) & \text { otherwise. }\end{cases}
$$

Here,

$$
H_{k}^{p_{i}}=G_{k}-a_{k}\left(p_{i}\right) \log r-A_{k}\left(p_{i}\right)-\lambda_{k}\left(p_{i}\right) r \cos \theta-\mu_{k}\left(p_{i}\right) r \sin \theta,
$$

and $\eta_{i}$ is a cut-off function which equals 1 in $B_{L \epsilon}\left(p_{i}\right)$, equals 0 in $B_{2 L \epsilon}^{c}\left(p_{i}\right)$. We may assume that

$$
\left|\nabla \eta_{i}\right| \leq \frac{1}{L \epsilon}
$$


Now we compute $\Phi_{0}(\phi)$. Firstly, we compute $\int_{\Sigma}\left|\nabla \phi_{1}\right|^{2} d V_{g}$ and $\int_{\Sigma}\left|\nabla \phi_{2}\right|^{2} d V_{g}$. Let $\Omega=\Sigma \backslash\left(B_{L \epsilon}\left(p_{1}\right) \cup B_{L \epsilon}\left(p_{2}\right)\right)$. Then

$$
\begin{aligned}
\int_{\Sigma}\left|\nabla \phi_{1}\right|^{2} d V_{g}= & \int_{B_{L \epsilon}\left(p_{1}\right) \cup B_{L \epsilon}\left(p_{2}\right)}\left|\nabla \phi_{1}\right|^{2} d x d y+\int_{\Omega}\left|\nabla G_{1}\right|^{2} d V_{g} \\
& -2 \sum_{i=1,2} \int_{\Sigma} \nabla G_{1} \nabla \eta_{i} H_{1}^{p_{i}} d V_{g}+\sum_{i=1,2} \int_{\Sigma}\left|\nabla \eta_{i} H_{1}^{p_{i}}\right|^{2} d V_{g} .
\end{aligned}
$$

It is clear that we have

$$
\int_{B_{L \epsilon}\left(p_{2}\right)}\left|\nabla \phi_{1}\right|^{2} d V_{g}=\frac{1}{4} \int_{B_{L}}|\nabla w|^{2} d x d y+\pi(L \epsilon)^{2}\left(\lambda_{1}^{2}\left(p_{2}\right)+\mu_{1}^{2}\left(p_{2}\right)\right),
$$

and

$$
\int_{B_{L \epsilon}\left(p_{1}\right)}\left|\nabla \phi_{1}\right|^{2} d V_{g}=\int_{B_{L}}|\nabla w|^{2} d x d y+\pi(L \epsilon)^{2}\left(\lambda_{1}^{2}\left(p_{1}\right)+\mu_{1}^{2}\left(p_{1}\right)\right) .
$$

Calculating directly and using the fact that $\int_{0}^{2 \pi} h d \theta=0$, we obtain,

$$
\begin{aligned}
\int_{\Sigma} \nabla G_{1} \nabla \eta_{1} H_{1}^{p_{1}} d V_{g}= & -\int_{\partial B_{L \epsilon}\left(p_{1}\right)} \frac{\partial G_{1}}{\partial n} H_{1}^{p_{1}} d S_{g}-4 \pi \int_{B_{2 L \epsilon \backslash B_{L \epsilon}}} \eta_{1} H_{1}^{p_{1}} d V_{g} \\
= & -\int_{0}^{2 \pi}\left(-\frac{4}{r}+\lambda_{1}\left(p_{1}\right) \cos \theta+\mu_{1}\left(p_{1}\right) \sin \theta+O(r)\right) \\
& \times\left(\alpha_{1}\left(p_{1}\right) r^{2} \cos ^{2} \theta+\beta_{1}\left(p_{1}\right) r^{2} \sin ^{2} \theta+h+O\left(r^{4}\right)\right) r d \theta \\
= & 4 \pi\left(\alpha_{1}\left(p_{1}\right)+\beta_{1}\left(p_{1}\right)\right)(L \epsilon)^{2}+O(L \epsilon)^{4} \\
= & 8 \pi^{2}(L \epsilon)^{2}+O(L \epsilon)^{4} .
\end{aligned}
$$

Similarly, we get

$$
\int_{\Sigma} \nabla G_{1} \nabla \eta_{2} H_{1}^{p_{2}} d V_{g}=-4 \pi^{2}(L \epsilon)^{2}+O(L \epsilon)^{4} .
$$

It is obvious that

$$
\int_{\Sigma}\left|\nabla \eta_{j} H_{i}^{p_{j}}\right|^{2} d V_{g}=\int_{B_{2 L \epsilon} \backslash B_{L \epsilon}\left(p_{j}\right)} O\left(r^{2}\right) d V_{g}=O\left((L \epsilon)^{4}\right) .
$$

Hence

$$
\begin{aligned}
\int_{\Sigma}\left|\nabla \phi_{1}\right|^{2} d V_{g}= & \frac{5}{4} \int_{B_{L}}|\nabla w|^{2} d x-8 \pi^{2}(L \epsilon)^{2}+\int_{\Omega}\left|\nabla G_{1}\right|^{2} d V_{g} \\
& +\pi(L \epsilon)^{2} \sum_{i=1,2}\left(\lambda_{1}^{2}\left(p_{i}\right)+\mu_{1}^{2}\left(p_{i}\right)\right)+O(L \epsilon)^{4}
\end{aligned}
$$


In the same way, we can show that

$$
\begin{aligned}
\int_{\Sigma}\left|\nabla \phi_{2}\right|^{2} d V_{g}= & \frac{5}{4} \int_{B_{L}}|\nabla w|^{2} d x-8 \pi^{2}(L \epsilon)^{2}+\int_{\Omega}\left|\nabla G_{2}\right|^{2} d V_{g} \\
& +\pi(L \epsilon)^{2} \sum_{i=1,2}\left(\lambda_{2}^{2}\left(p_{i}\right)+\mu_{2}^{2}\left(p_{i}\right)\right)+O(L \epsilon)^{4} .
\end{aligned}
$$

Next, we compute $\int_{\Sigma} \nabla \phi_{1} \nabla \phi_{2} d V_{g}$. We have

$$
\begin{aligned}
\int_{\Sigma} \nabla \phi_{1} \nabla \phi_{2} d V_{g}= & \sum_{i=1,2} \int_{B_{L \epsilon}\left(p_{i}\right)} \nabla \phi_{1} \nabla \phi_{2} d V_{g}+\int_{\Omega} \nabla G_{1} \nabla G_{2} d V_{g} \\
& -\int_{\Sigma} \nabla G_{1} \nabla \eta_{1} H_{2}^{p_{1}} d V_{g}-\int_{\Sigma} \nabla G_{2} \nabla \eta_{1} H_{1}^{p_{1}} d V_{g} \\
& -\int_{\Sigma} \nabla G_{1} \nabla \eta_{2} H_{2}^{p_{2}} d V_{g}-\int_{\Sigma} \nabla G_{2} \nabla \eta_{2} H_{1}^{p_{2}} d V_{g} \\
& +\sum_{i=1,2} \int_{\Sigma} \nabla \eta_{i} H_{1}^{p_{i}} \nabla \eta_{i} H_{2}^{p_{i}} d V_{g} \\
= & -\int_{B_{L}}|\nabla w|^{2} d x+\pi(L \epsilon)^{2} \sum_{i=1,2}\left(\lambda_{1}\left(p_{i}\right) \lambda_{2}\left(p_{i}\right)+\mu_{1}\left(p_{i}\right) \mu_{2}\left(p_{i}\right)\right) \\
& -8 \pi(L \epsilon)^{2}+\int_{\Omega} \nabla G_{1} \nabla G_{2} d V_{g}+O\left((L \epsilon)^{4}\right) .
\end{aligned}
$$

Then we calculate $\int_{\Omega}\left(\left|\nabla G_{1}\right|^{2}+\left|\nabla G_{2}\right|^{2}+\nabla G_{1} \nabla G_{2}\right) d V_{g}$. We have

$$
\begin{aligned}
& \int_{\Omega}\left(\left|\nabla G_{1}\right|^{2}+\left|\nabla G_{2}\right|^{2}+\nabla G_{1} \nabla G_{2}\right) d V_{g} \\
& =\int_{\Omega}\left(\left|\nabla G_{1}\right|^{2}+\left|\nabla G_{2}\right|^{2}+\frac{\nabla G_{1} \nabla G_{2}+\nabla G_{2} \nabla G_{1}}{2}\right) d V_{g} \\
& =-\int_{\partial B_{L \epsilon}\left(p_{1}\right)+\partial B_{L \epsilon}\left(p_{2}\right)}\left(G_{1} \frac{\partial G_{1}}{\partial n}+G_{2} \frac{\partial G_{2}}{\partial n}+\frac{G_{1} \frac{\partial G_{2}}{\partial n}+G_{2} \frac{\partial G_{1}}{\partial n}}{2}\right) d S_{g} \\
& \quad+6 \pi \int_{B_{L \epsilon\left(p_{1}\right)}+B_{L \epsilon}\left(p_{2}\right)}\left(G_{1}+G_{2}\right) d V_{g} .
\end{aligned}
$$

Lemma 5.2. For any $k, m, i=1,2$, we have

$$
\begin{aligned}
\int_{\partial B_{r}\left(p_{i}\right)} G_{k} \frac{\partial G_{m}}{\partial n} d S_{g}= & 2 \pi a_{k}\left(p_{i}\right) a_{m}\left(p_{i}\right) \log r+2 \pi^{2} a_{k}\left(p_{i}\right) r^{2} \\
& +\pi r^{2}\left(\lambda_{k}\left(p_{i}\right) \lambda_{m}\left(p_{i}\right)+\mu_{k}\left(p_{i}\right) \mu_{m}\left(p_{i}\right)\right) \\
& +2 \pi a_{k}\left(p_{i}\right) A_{m}\left(p_{i}\right)+4 \pi^{2} r^{2} A_{m}\left(p_{i}\right) \\
& +4 \pi^{2} r^{2} a_{m}\left(p_{i}\right) \log r+O\left(r^{4} \log r\right) .
\end{aligned}
$$


Proof. Since $\int_{0}^{2 \pi} h(r, \theta) d \theta=\int_{0}^{2 \pi} \frac{\partial h}{\partial r}(r, \theta) d \theta=0$, we have

$$
\begin{aligned}
\int_{\partial B_{r}\left(p_{i}\right)} G_{k} \frac{\partial G_{m}}{\partial n} d S_{g}= & \int_{0}^{2 \pi}\left(\frac{a_{k}\left(p_{i}\right)}{r}+\lambda_{k}\left(p_{i}\right) \cos \theta+\mu_{k}\left(p_{i}\right) \sin \theta+2 r \alpha_{k}\left(p_{i}\right) \cos ^{2} \theta\right. \\
& \left.+2 r \beta_{k}\left(p_{i}\right) \sin ^{2} \theta+2 r \gamma_{k}\left(p_{i}\right) \sin \theta \cos \theta\right)\left(a_{m}\left(p_{i}\right) \log r\right. \\
& +A_{m}\left(p_{i}\right)+\lambda_{m}\left(p_{i}\right) r \cos \theta+\mu_{m}\left(p_{i}\right) r \sin \theta+r^{2} \alpha_{m}\left(p_{i}\right) \cos ^{2} \theta \\
& \left.+r^{2} \beta_{m}\left(p_{i}\right) \sin ^{2} \theta+r^{2} \gamma_{m}\left(p_{i}\right) \sin \theta \cos \theta\right) r d \theta \\
& +O\left(r^{4} \log r\right) \\
= & 2 \pi a_{k}\left(p_{i}\right) a_{m}\left(p_{i}\right) \log r+\pi\left(\alpha_{m}\left(p_{i}\right)+\beta_{m}\left(p_{i}\right)\right) a_{k}\left(p_{i}\right) r^{2} \\
& +\pi r^{2}\left(\lambda_{k}\left(p_{i}\right) \lambda_{m}\left(p_{i}\right)+\mu_{k}\left(p_{i}\right) \mu_{m}\left(p_{i}\right)\right)+2 \pi a_{k}\left(p_{i}\right) A_{m}\left(p_{i}\right) \\
& +\left(2 \pi r^{2} A_{m}\left(p_{i}\right)+2 \pi r^{2} a_{m}\left(p_{i}\right) \log r\right)\left(\alpha_{k}\left(p_{i}\right)+\beta_{k}\left(p_{i}\right)\right) \\
& +O\left(r^{4} \log r\right) .
\end{aligned}
$$

Then

$$
\begin{aligned}
\int_{\partial B_{r}\left(p_{1}\right)} G_{1} \frac{\partial G_{1}}{\partial n} d S_{g}= & 32 \pi \log r-8 \pi^{2} r^{2}+\pi r^{2}\left(\lambda_{1}^{2}\left(p_{1}\right)+\mu_{1}^{2}\left(p_{1}\right)\right) \\
& -8 \pi A_{1}\left(p_{1}\right)+4 \pi^{2} r^{2} A_{1}\left(p_{1}\right)-16 \pi^{2} r^{2} \log r+O\left(r^{4} \log r\right) . \\
\int_{\partial B_{r}\left(p_{1}\right)} G_{2} \frac{\partial G_{2}}{\partial n} d S_{g}= & 8 \pi \log r+4 \pi^{2} r^{2}+\pi r^{2}\left(\lambda_{2}^{2}\left(p_{1}\right)+\mu_{2}^{2}\left(p_{1}\right)\right) \\
& +4 \pi A_{2}\left(p_{1}\right)+4 \pi^{2} r^{2} A_{2}\left(p_{1}\right)+8 \pi^{2} r^{2} \log r+O\left(r^{4} \log r\right) . \\
\int_{\partial B_{r}\left(p_{1}\right)} G_{1} \frac{\partial G_{2}}{\partial n} d S_{g}= & -16 \pi \log r-8 \pi^{2} r^{2}+\pi r^{2}\left(\lambda_{1}\left(p_{1}\right) \lambda_{2}\left(p_{1}\right)+\mu_{1}\left(p_{1}\right) \mu_{2}\left(p_{1}\right)\right) \\
& -8 \pi A_{2}\left(p_{1}\right)+4 \pi^{2} r^{2} A_{2}\left(p_{1}\right)+8 \pi^{2} r^{2} \log r+O\left(r^{4} \log r\right) . \\
\int_{\partial B_{r}\left(p_{1}\right)} G_{2} \frac{\partial G_{1}}{\partial n} d S_{g}= & -16 \pi \log r+4 \pi^{2} r^{2}+\pi r^{2}\left(\lambda_{2}\left(p_{1}\right) \lambda_{1}\left(p_{1}\right)+\mu_{2}\left(p_{1}\right) \mu_{1}\left(p_{1}\right)\right) \\
+ & +4 \pi A_{1}\left(p_{1}\right)+4 \pi^{2} r^{2} A_{1}\left(p_{1}\right)-16 \pi^{2} r^{2} \log r+O\left(r^{4} \log r\right) .
\end{aligned}
$$


Hence

$$
\begin{aligned}
& \int_{\Omega}\left(\left|\nabla G_{1}\right|^{2}+\left|\nabla G_{2}\right|^{2}\right) d V_{g} \\
& =-\left(80 \pi \log L \epsilon-8 \pi^{2}(L \epsilon)^{2}+\pi(L \epsilon)^{2} \sum_{i, j=1,2}\left(\lambda_{i}^{2}(j)+\mu_{i}^{2}(j)\right)\right. \\
& \quad-8 \pi A_{1}\left(p_{1}\right)-8 \pi A_{2}\left(p_{2}\right)+4 \pi^{2}(L \epsilon)^{2}\left(A_{1}\left(p_{1}\right)+A_{2}\left(p_{2}\right)+A_{2}\left(p_{1}\right)+A_{1}\left(p_{2}\right)\right) \\
& \left.-16 \pi^{2}(L \epsilon)^{2} \log L \epsilon+4 \pi A_{2}\left(p_{1}\right)+4 \pi A_{1}\left(p_{2}\right)\right)+4 \pi \int_{\Omega} G_{1} d V_{g}+4 \pi \int_{\Omega} G_{2} d V_{g} \\
& +O\left((L \epsilon)^{4} \log L \epsilon\right),
\end{aligned}
$$

and

$$
\begin{aligned}
& \int_{\Omega} \nabla G_{1} \nabla G_{2} d V_{g} \\
& =-\left(-32 \log L \epsilon-4 \pi^{2}(L \epsilon)^{2}+\pi(L \epsilon)^{2} \sum_{i \neq j}\left(\lambda_{i}\left(p_{j}\right) \lambda_{j}\left(p_{i}\right)+\mu_{i}\left(p_{j}\right) \mu_{j}\left(p_{i}\right)\right)\right. \\
& \quad-4 \pi A_{2}\left(p_{1}\right)-4 \pi A_{1}\left(p_{2}\right)+2 \pi A_{1}\left(p_{1}\right)+2 \pi A_{2}\left(p_{2}\right) \\
& \left.\quad+2 \pi^{2}(L \epsilon)^{2}\left(A_{1}\left(p_{1}\right)+A_{1}\left(p_{2}\right)+A_{2}\left(p_{1}\right)+A_{2}\left(p_{2}\right)\right)-8 \pi^{2}(L \epsilon)^{2} \log L \epsilon\right) \\
& \quad+2 \pi \sum_{i=1,2} \int_{B_{L \epsilon}\left(p_{i}\right)}\left(G_{1}+G_{2}\right) d V_{g}+O\left((L \epsilon)^{4} \log L \epsilon\right) .
\end{aligned}
$$

It is easy to check that

$$
\begin{aligned}
6 \pi \int_{B_{L \epsilon}\left(p_{1}\right)+B_{L \epsilon}\left(p_{2}\right)}\left(G_{1}+G_{2}\right) d V_{g}= & 6 \pi^{2}(L \epsilon)^{2}\left(\sum_{i, j=1,2} A_{i}\left(p_{j}\right)\right)-24 \pi^{2}(L \epsilon)^{2} \log L \epsilon \\
& +12 \pi^{2}(L \epsilon)^{2}+O\left((L \epsilon)^{4} \log L \epsilon\right) .
\end{aligned}
$$

So, we get

$$
\begin{aligned}
\int_{\Sigma}\left(\left|\nabla \phi_{1}\right|^{2}+\left|\nabla \phi_{2}\right|^{2}+\nabla \phi_{1} \nabla \phi_{2}\right) d V_{g}= & \frac{3}{2} \int_{B_{L}}|\nabla w|^{2} d x d y-48 \pi \log L \epsilon+6 \pi A_{1}\left(p_{1}\right) \\
& +6 \pi A_{2}\left(p_{2}\right)+O\left((L \epsilon)^{4} \log L \epsilon\right) .
\end{aligned}
$$


We calculate $\int_{\Sigma}\left(\phi_{1}+\phi_{2}\right) d V_{g}$. We have

$$
\begin{aligned}
\int_{\Sigma} \phi_{1} d V_{g}= & \epsilon^{2} \int_{B_{L}} w e^{\varphi(\epsilon x, \epsilon y)} d x d y-\int_{\Sigma}\left(\eta_{1} H_{1}^{p_{1}}+\eta_{2} H_{1}^{p_{2}}\right) d V_{g} \\
& +\left(4 \log L \epsilon-2 \log \left(1+\pi L^{2}\right)-A_{1}\left(p_{1}\right)\right) \\
& \times\left(1-\int_{B_{L \epsilon}\left(p_{1}\right)} d V_{g}\right)+2 \log L \epsilon \int_{B_{L \epsilon}\left(p_{2}\right)} d V_{g} \\
& +A_{1}\left(p_{2}\right) \int_{B_{L \epsilon}\left(p_{2}\right)} d V_{g}-\int_{B_{L \epsilon}\left(p_{1}\right)+B_{L \epsilon}\left(p_{2}\right)} G_{1} \\
& -\epsilon^{2} \int_{B_{L}} \frac{w+2 \log \left(1+\pi L^{2}\right)}{2} d V_{g}\left(\lambda_{1}\left(p_{i}\right) x+\mu_{1}\left(p_{i}\right) y\right) d V_{g} . \\
& +\sum_{i=1,2} \int_{B_{L \epsilon}\left(p_{i}\right)}
\end{aligned}
$$

Since

$$
\begin{aligned}
\int_{B_{L \epsilon}\left(p_{1}\right)+B_{L \epsilon}\left(p_{2}\right)} G_{1} d V_{g}= & \int_{0}^{L \epsilon}\left(-2 \log r+A_{1}\left(p_{1}\right)+A_{1}\left(p_{2}\right)\right) 2 \pi r d r \\
& +O\left((L \epsilon)^{4} \log L \epsilon\right) \\
= & -2 \pi(L \epsilon)^{2} \log L \epsilon+\pi(L \epsilon)^{2} \\
& +\left(A_{1}\left(p_{1}\right)+A_{1}\left(p_{2}\right)\right) \pi(L \epsilon)^{2}+O\left((L \epsilon)^{4} \log L \epsilon\right),
\end{aligned}
$$

we have

$$
\begin{aligned}
\int_{\Sigma} \phi_{1} d V_{g}= & \frac{\epsilon^{2}}{2} \int_{B_{L}} w e^{\varphi(\epsilon x, \epsilon y)} d x d y+4 \log L \epsilon+\pi(L \epsilon)^{2} \log \left(1+\pi L^{2}\right) \\
& -\pi(L \epsilon)^{2}-A_{1}\left(p_{1}\right)-2 \log \left(1+\pi L^{2}\right)+O\left((L \epsilon)^{4} \log L \epsilon\right) .
\end{aligned}
$$

Similarly, we have

$$
\begin{aligned}
\int_{\Sigma} \phi_{2} d V_{g}= & \frac{\epsilon^{2}}{2} \int_{B_{L}} w e^{\varphi(\epsilon x, \epsilon y)} d x d y+4 \log L \epsilon+\pi(L \epsilon)^{2} \log \left(1+\pi L^{2}\right) \\
& -\pi(L \epsilon)^{2}-A_{2}\left(p_{2}\right)-2 \log \left(1+\pi L^{2}\right)+O\left((L \epsilon)^{4} \log L \epsilon\right) .
\end{aligned}
$$

Moreover, we have

$$
\int_{B_{L}} \omega e^{\varphi(\epsilon x, \epsilon y)} d x d y=2 \pi L^{2}-2 \log \left(1+\pi L^{2}\right)-2 \pi L^{2} \log \left(1+\pi L^{2}\right)+O\left(L^{2} \epsilon^{2} \log L\right),
$$


hence, we get

$$
\begin{aligned}
\int_{\Sigma}\left(\phi_{1}+\phi_{2}\right) d V_{g}= & -A_{1}\left(p_{1}\right)-A_{2}\left(p_{2}\right)+8 \log L \epsilon-4 \log \left(1+\pi L^{2}\right) \\
& -2 \epsilon^{2} \log \left(1+\pi L^{2}\right)+O\left((L \epsilon)^{4} \log L \epsilon\right) .
\end{aligned}
$$

We denote $B\left(p_{j}\right)=\frac{\left(b_{1}\left(p_{j}\right)+\lambda_{1}\left(p_{j}\right)\right)^{2}+\left(b_{2}\left(p_{j}\right)+\lambda_{2}\left(p_{j}\right)\right)^{2}}{4}$ and $M_{i}=\frac{-\frac{K\left(p_{i}\right)}{2}+B\left(p_{i}\right)}{\pi}$. Then we have

$$
\begin{aligned}
& \int_{B_{L \epsilon}\left(p_{1}\right)} e^{\phi_{1}} d V_{g} \\
= & \epsilon^{2} \int_{B_{L}} \frac{e^{\left(b_{1}\left(p_{1}\right)+\lambda_{1}\left(p_{1}\right)\right) \epsilon x+\left(b_{2}\left(p_{1}\right)+\lambda_{2}\left(p_{1}\right)\right) \epsilon y+c_{1}\left(p_{1}\right) \epsilon^{2} x^{2}+c_{2}\left(p_{1}\right) \epsilon^{2} y^{2}+c_{12} \epsilon^{2} x y+O\left((r \epsilon)^{3}\right)}}{\left(1+\pi r^{2}\right)^{2}} d x d y \\
= & \epsilon^{2} \int_{0}^{L} \frac{1+\epsilon^{2} \pi M_{i} r^{2}+O\left(\epsilon^{3} r^{3}\right)}{\left(1+\pi r^{2}\right)^{2}} 2 \pi r d r \\
= & \epsilon^{2}\left(1-\epsilon^{2} M_{i}\right) \frac{\pi L^{2}}{1+\pi L^{2}}+\epsilon^{4} M_{i} \log \left(1+\pi L^{2}\right)+\epsilon^{2} O\left(\epsilon^{3} \log L\right) \\
= & \epsilon^{2}\left(1-\frac{1}{1+\pi L^{2}}+\epsilon^{2} M_{i} \log \left(1+\pi L^{2}\right)+O\left(\epsilon^{2}\right)+O\left(\epsilon^{3} \log L\right)\right),
\end{aligned}
$$

and we also have

$$
\begin{aligned}
\int_{B_{\delta}\left(p_{1}\right) \backslash B_{L \epsilon\left(p_{1}\right)}} e^{\phi_{1}} d V_{g} \\
=\frac{(L \epsilon)^{4}}{\left(1+\pi L^{2}\right)^{2}} \int_{L \epsilon}^{\delta} e^{-4 \log r+\left(\lambda_{1}\left(p_{1}\right)+b_{1}\left(p_{1}\right)\right) x+\left(\mu_{1}\left(p_{1}\right)+b_{2}\left(p_{1}\right)\right) y} \\
\quad \times e^{\left(c_{1}\left(p_{1}\right)+\alpha_{1}\left(p_{1}\right)\right) x^{2}+\left(c_{2}\left(p_{1}\right)+\beta_{1}\left(p_{1}\right)\right) y^{2}+\left(c_{12}\left(p_{1}\right)+\gamma_{1}\right) x y+\left(1-\eta_{1}\right) H_{1}^{p_{1}}+O\left(r^{3}\right)} 2 \pi r d r \\
=\epsilon^{2}\left(\frac{\pi L^{2}}{\left(1+\pi L^{2}\right)^{2}}-2\left(M_{1}+1\right) \epsilon^{2} \log L \epsilon+O\left(\epsilon^{2}\right)+O\left(\frac{1}{L^{4}}\right)\right) .
\end{aligned}
$$

Since $G_{1}$ is bounded above, outsid $B_{\delta}\left(p_{1}\right)$, we have

$$
\int_{\Sigma \backslash B_{\delta}} e^{\phi_{1}}=O\left(\epsilon^{4}\right) .
$$

Noting that $\frac{\pi L^{2}}{\left(1+\pi L^{2}\right)^{2}}-\frac{1}{1+\pi L^{2}}=O\left(\frac{1}{L^{4}}\right)$, we get

$$
\begin{gathered}
\log \int_{\Sigma} e^{\phi_{1}} d V_{g}=\log \epsilon^{2}+\epsilon^{2} M_{1} \log \left(1+\pi L^{2}\right)-2 \epsilon^{2}\left(M_{1}+1\right) \log L \epsilon \\
O\left(\epsilon^{2}\right)+O\left(\frac{1}{L^{4}}\right) .
\end{gathered}
$$


In the same way, we can get

$$
\begin{aligned}
\log \int_{\Sigma} e^{\phi_{2}} d V_{g}= & \log \epsilon^{2}+\epsilon^{2} M_{2} \log \left(1+\pi L^{2}\right)-2 \epsilon^{2}\left(M_{2}+1\right) \log L \epsilon \\
& +O\left(\epsilon^{2}\right)+O\left(\frac{1}{L^{4}}\right) .
\end{aligned}
$$

It follows from (5.1), (5.2), (5.3) and (5.4) that

$$
\begin{aligned}
\Phi_{0}(\phi)= & \frac{1}{2} \int_{B_{L}}|\nabla w|^{2} d x d y+16 \log L \epsilon-2 \pi\left(A_{1}\left(p_{1}\right)+A_{2}\left(p_{2}\right)\right) \\
& -16 \pi \log \left(1+\pi L^{2}\right)-8 \pi \log \epsilon^{2}-8 \pi \epsilon^{2} \log \left(1+\pi L^{2}\right) \\
& -4 \pi \epsilon^{2}\left(\left(M_{1}+M_{2}\right) \log \left(1+\pi L^{2}\right)-2\left(M_{1}+M_{2}+2\right) \log L \epsilon\right) \\
& +O\left(\frac{1}{L^{4}}\right)+O\left(\epsilon^{2}\right)+O\left((L \epsilon)^{4} \log L \epsilon\right)+O\left(\epsilon^{3} \log L\right) \\
= & -8 \pi \log \frac{1+\pi L^{2}}{L^{2}}-8 \pi \frac{\pi L^{2}}{1+\pi L^{2}}-2 \pi\left(A_{1}\left(p_{1}\right)+A_{2}\left(p_{2}\right)\right) \\
& -4 \pi \epsilon^{2}\left(M_{1}+M_{2}+2\right)\left(\log \left(1+\pi L^{2}\right)-2 \log L \epsilon\right) \\
& +O\left(\frac{1}{L^{4}}\right)+O\left(\epsilon^{2}\right)+O\left((L \epsilon)^{4} \log L \epsilon\right)+O\left(\epsilon^{3} \log L\right) \\
= & -8 \pi \log \pi-8 \pi-2 \pi\left(A_{1}\left(p_{1}\right)+A_{2}\left(p_{2}\right)\right) \\
& -4 \pi\left(M_{1}+M_{2}+2\right) \epsilon^{2}\left(\log \left(1+\pi L^{2}\right)-2 \log L \epsilon\right) \\
& +O\left(\frac{1}{L^{4}}\right)+O\left(\epsilon^{2}\right)+O\left((L \epsilon)^{4} \log L \epsilon\right)+O\left(\epsilon^{3} \log L\right) .
\end{aligned}
$$

Under assumption (1.1) we have $M_{1}+M_{2}+2>0$. Leting $L^{4} \epsilon^{2}=\frac{1}{\log (-\log \epsilon)}$, we get

$$
\begin{aligned}
\Phi_{0}(\phi)= & -8 \pi \log \pi-8 \pi-2 \pi\left(A_{1}\left(p_{1}\right)+A_{2}\left(p_{2}\right)\right) \\
& -4 \pi\left(M_{1}+M_{2}+2\right) \epsilon^{2}\left(-\log \epsilon^{2}\right)+o\left(\epsilon^{2}\left(-\log \epsilon^{2}\right)\right) .
\end{aligned}
$$

Then for sufficiently small $\epsilon$ we have

$$
\Phi_{0}(\phi)<-8 \pi \log \pi-8 \pi-2 \pi\left(A_{1}\left(p_{1}\right)+A_{2}\left(p_{2}\right)\right) .
$$

This proves our claim.

\section{Test functions for Case 2}

Assuming that (1.4) holds on $\Sigma$, we will construct a function $\phi=\left(\phi_{1}, \phi_{2}\right) \in$ $H^{1,2}(M) \times H^{1,2}(M)$, such that

$$
\Phi_{0}(\phi)<-4 \pi \log \pi-2 \pi A_{1}(p)+2 \pi \int G_{2} d V_{g} .
$$


Let $(\Omega ;(x, y))$ be an isothermal coordinate system around $p$. We assume that near $p$ $G_{k}=a_{k} \log r+A_{k}(p)+\lambda_{k} x+\mu_{k} y+\alpha_{k} x^{2}+\beta_{k} y^{2}+\gamma_{k} x y+h_{k}(x, y)+O\left(r^{4}\right)$.

We have $a_{1}(p)=-4$, and $a_{2}\left(p_{1}\right)=2$. Moreover we assume that

$$
\left.g\right|_{\Omega}=e^{\varphi}\left(d x^{2}+d y^{2}\right),
$$

and

$$
\varphi=b_{1} x+b_{2} y+c_{1} x^{2}+c_{2} y^{2}+c_{12} x y+O\left(r^{3}\right) .
$$

Similarly to Case 1 , there hold

$$
\alpha_{k}+\beta_{k}=2 \pi, \quad i=1,2 .
$$

We choose

$$
\phi_{1}= \begin{cases}w\left(\frac{x}{\epsilon}\right)+\lambda_{1} r \cos \theta+\mu_{1} r \sin \theta & x \in B_{L \epsilon}(p) \\ G_{1}-\eta H_{1}+4 \log L \epsilon-2 \log \left(1+\pi L^{2}\right)-A_{1}(p) & x \in B_{2 L \epsilon} \backslash B_{L \epsilon}(p) \\ G_{1}+4 \log L \epsilon-2 \log \left(1+\pi L^{2}\right)-A_{1}\left(p_{1}\right) & \text { otherwise, }\end{cases}
$$

and

$$
\phi_{2}= \begin{cases}-\frac{w\left(\frac{x}{\epsilon}\right)+2 \log \left(1+\pi L^{2}\right)}{2}+2 \log L \epsilon & \\ \quad+\lambda_{2} r \cos \theta+\mu_{2} r \sin \theta+A_{2}(p) & x \in B_{L \epsilon}(p) \\ G_{2}-\eta H_{2} & x \in B_{2 L \epsilon} \backslash B_{L \epsilon}(p) \\ G_{2} & \text { otherwise. }\end{cases}
$$

Here

$$
H_{k}=G_{k}-a_{k} \log r-A_{k}+\lambda_{k} r \cos \theta+\mu_{k} r \sin \theta
$$

and $\eta_{i}$ is a cut-off function which equals 1 in $B_{L \epsilon}(p)$, equals 0 in $B_{2 L \epsilon}^{c}(p)$.

Let $\Omega=\Sigma \backslash B_{L \epsilon}(p)$. By an argument similar that used in Section 5, we can derive that

$$
\begin{aligned}
\int_{\Sigma}\left|\nabla \phi_{1}\right|^{2} d V_{g}= & \int_{B_{L \epsilon}(p)}\left|\nabla \phi_{1}\right|^{2} d x d y+\int_{\Omega}\left|\nabla G_{1}\right|^{2} d V_{g} \\
& -2 \int_{\Sigma} \nabla G_{1} \nabla \eta H_{1} d V_{g}+\int_{\Sigma}\left|\nabla \eta H_{1}\right|^{2} d V_{g} \\
= & \int_{B_{L}}|\nabla w|^{2} d x d y-16 \pi(L \epsilon)^{2}+\pi(L \epsilon)^{2}\left(\lambda_{1}^{2}+\mu_{1}^{2}\right)+O\left((L \epsilon)^{4}\right),
\end{aligned}
$$




$$
\begin{aligned}
\int_{\Sigma}\left|\nabla \phi_{2}\right|^{2} d V_{g}= & \int_{B_{L \epsilon}(p)}\left|\nabla \phi_{1}\right|^{2} d x d y+\int_{\Omega}\left|\nabla G_{2}\right|^{2} d V_{g} \\
& -2 \int_{\Sigma} \nabla G_{2} \nabla \eta_{2} H_{2} d V_{g}+\int_{\Sigma}\left|\nabla \eta H_{2}\right|^{2} d V_{g} \\
= & \frac{1}{4} \int_{B_{L}}|\nabla w|^{2} d x d y+8 \pi(L \epsilon)^{2}+\pi(L \epsilon)^{2}\left(\lambda_{2}^{2}+\mu_{2}^{2}\right)+O\left((L \epsilon)^{4}\right),
\end{aligned}
$$

and

$$
\begin{aligned}
\int_{\Sigma} \nabla \phi_{1} \nabla \phi_{2} d V_{g}= & \int_{\Omega} \nabla G_{1} \nabla G_{2} d V_{g}+\int_{B_{L \epsilon}(p)} \nabla \phi_{1} \nabla \phi_{2} d V_{g} \\
& -\int \nabla G_{1} \nabla \eta H_{2} d V_{g}-\int^{\nabla} \nabla G_{2} \nabla \eta H_{1} d V_{g}+\int_{\Sigma} \nabla \eta H_{1} \nabla \eta H_{2} d V_{g} \\
= & -\frac{1}{2} \int_{B_{L}}|\nabla w|^{2} d x d y+\pi(L \epsilon)^{2}\left(\lambda_{1} \lambda_{2}+\mu_{1} \mu_{2}\right) \\
& -4 \pi(L \epsilon)^{2}+\int_{\Omega} \nabla G_{1} \nabla G_{2} d V_{g}+O\left((L \epsilon)^{4}\right) .
\end{aligned}
$$

Note that

$$
\begin{aligned}
& \int_{\Omega}\left(\left|\nabla G_{1}\right|^{2}+\left|\nabla G_{2}\right|^{2}+\nabla G_{1} \nabla G_{2}\right) d V_{g} \\
& =\int_{\Omega}\left(\left|\nabla G_{1}\right|^{2}+\left|\nabla G_{2}\right|^{2}+\frac{\nabla G_{1} \nabla G_{2}+\nabla G_{2} \nabla G_{1}}{2}\right) d V_{g} \\
& =-\int_{\partial B_{L \epsilon}(p)}\left(G_{1} \frac{\partial G_{1}}{\partial n}+G_{2} \frac{\partial G_{2}}{\partial n}+\frac{G_{1} \frac{\partial G_{2}}{\partial n}+G_{2} \frac{\partial G_{1}}{\partial n}}{2}\right) d S_{g} \\
& \quad+6 \pi \int_{B_{L \epsilon(p)}}\left(G_{1}+G_{2}\right)-6 \pi \int_{\Sigma} G_{2} d V_{g} .
\end{aligned}
$$

Applying Lemma 5.2, we get

$$
\begin{aligned}
\int_{\partial B_{r}(p)} G_{1} \frac{\partial G_{1}}{\partial n} d S_{g}= & 32 \pi \log r-8 \pi^{2} r^{2}+\pi r^{2}\left(\lambda_{1}^{2}+\mu_{1}^{2}\right) \\
& -8 \pi A_{1}(p)+4 \pi^{2} r^{2} A_{1}(p)-16 \pi^{2} r^{2} \log r+O\left(r^{4} \log r\right), \\
\int_{\partial B_{r}(p)} G_{2} \frac{\partial G_{2}}{\partial n} d S_{g}= & 8 \pi \log r+4 \pi^{2} r^{2}+\pi r^{2}\left(\lambda_{2}^{2}+\mu_{2}^{2}\right) \\
& +4 \pi A_{2}(p)+4 \pi^{2} r^{2} A_{2}(p)+8 \pi^{2} r^{2} \log r+O\left(r^{4} \log r\right), \\
\int_{\partial B_{r}(p)} G_{1} \frac{\partial G_{2}}{\partial n} d S_{g}= & -16 \pi \log r-8 \pi^{2} r^{2}+\pi r^{2}\left(\lambda_{1} \lambda_{2}+\mu_{1} \mu_{2}\right) \\
& -8 \pi A_{2}(p)+4 \pi^{2} r^{2} A_{2}(p)+8 \pi^{2} r^{2} \log r+O\left(r^{4} \log r\right),
\end{aligned}
$$




$$
\begin{aligned}
\int_{\partial B_{r}(p)} G_{2} \frac{\partial G_{1}}{\partial n} d S_{g}= & -16 \pi \log r+4 \pi^{2} r^{2}+\pi r^{2}\left(\lambda_{2} \lambda_{1}+\mu_{2} \mu_{1}\right) \\
& +4 \pi A_{1}(p)+4 \pi^{2} r^{2} A_{1}(p)-16 \pi^{2} r^{2} \log r+O\left(r^{4} \log r\right) .
\end{aligned}
$$

Noticing that

$$
\begin{aligned}
6 \pi \int_{B_{L \epsilon}(p)}\left(G_{1}+G_{2}\right) d V_{g}= & 6 \pi^{2}(L \epsilon)^{2}\left(A_{1}(p)+A_{2}(p)\right) \\
& -12 \pi^{2}(L \epsilon)^{2} \log L \epsilon+6 \pi^{2}(L \epsilon)^{2}+O\left((L \epsilon)^{4} \log L \epsilon\right),
\end{aligned}
$$

we get

$$
\begin{aligned}
& \int_{\Sigma}\left(\left|\nabla \phi_{1}\right|^{2}+\left|\nabla \phi_{2}\right|^{2}+\nabla \phi_{1} \nabla \phi_{2}\right) d V_{g} \\
& =\frac{3}{4} \int_{B_{L}}|\nabla w|^{2} d x d y-24 \pi \log L \epsilon \\
& \quad-6 \pi A_{1}\left(p_{1}\right)-6 \pi \int G_{2} d V_{g}+O\left((L \epsilon)^{4} \log L \epsilon\right) .
\end{aligned}
$$

We have

$$
\begin{aligned}
\int_{\Sigma} \phi_{1} d V_{g}= & \epsilon^{2} \int_{B_{L}} w d V_{g}-\int_{\Sigma} \eta H_{1} d V_{g}+\left(4 \log L \epsilon-2 \log \left(1+\pi L^{2}\right)\right. \\
& \left.-A_{1}(p)\right)\left(1-\int_{B_{L \epsilon}(p)} d V_{g}\right)+\int_{B_{L \epsilon}(p)}\left(\lambda_{1} x+\mu_{1} y\right) d V_{g} \\
& -\int_{B_{L \epsilon}(p)} G_{1} d V_{g}+O\left((L \epsilon)^{4} \log L \epsilon\right) \\
= & \epsilon^{2} \int_{B_{L}} w e^{\varphi(\epsilon x, \epsilon y)} d x d y+4 \log L \epsilon+2 \pi(L \epsilon)^{2} \log \left(1+\pi L^{2}\right) \\
& -2 \pi(L \epsilon)^{2}-A_{1}(p)-2 \log \left(1+\pi L^{2}\right)+O\left((L \epsilon)^{4} \log L \epsilon\right) .
\end{aligned}
$$

Since

$$
\begin{aligned}
\int_{B_{L \epsilon}(p)} G_{2} d V_{g} & =\int_{0}^{L \epsilon}\left(2 \log r+A_{2}(p)\right) 2 \pi L \epsilon d r+O\left((L \epsilon)^{4} \log L \epsilon\right) \\
& =2 \pi r^{2} \log r-\pi r^{2}+\left(A_{1}(p) \pi r^{2}+O\left((L \epsilon)^{4} \log L \epsilon\right),\right.
\end{aligned}
$$


we can see that

$$
\begin{aligned}
\int_{\Sigma} \phi_{2} d V_{g}= & \int_{\Sigma} G_{2} d V_{g}-\int_{B_{L \epsilon}(p)} G_{2} d V_{g} \\
& -\int_{\Sigma} \eta \beta_{2} d V_{g}+\left(2 \log L \epsilon+A_{1}(p)\right) \int_{B_{L \epsilon}} d V_{g} \\
& -\epsilon^{2} \int_{B_{L}} \frac{w+2 \log \left(1+\pi L^{2}\right)}{2} e^{\varphi(\epsilon x, \epsilon y)} d x d y+O\left((L \epsilon)^{4} \log L \epsilon\right) \\
= & -\frac{\epsilon^{2}}{2} \int_{B_{L}} w e^{\varphi(\epsilon x, \epsilon y)} d x d y \\
& -\pi(L \epsilon)^{2} \log \left(1+\pi L^{2}\right)+\pi(L \epsilon)^{2}+O\left((L \epsilon)^{4} \log L \epsilon\right) .
\end{aligned}
$$

Hence,

$$
\begin{aligned}
\int\left(\phi_{1}+\phi_{2}\right) d V_{g}= & -A_{1}(p)+4 \log L \epsilon-\epsilon^{2} \log \left(1+\pi L^{2}\right) \\
& -2 \log \left(1+\pi L^{2}\right)+O\left((L \epsilon)^{4} \log L \epsilon\right) .
\end{aligned}
$$

Setting $B(p)=\frac{\left(b_{1}+\lambda_{1}\right)^{2}+\left(b_{2}+\lambda_{2}\right)^{2}}{4}$ and $M=\frac{-\frac{K(p)}{2}+B(p)}{\pi}$, we have

$$
\begin{aligned}
\log \int_{\Sigma} e^{\phi_{1}} d V_{g}= & \log \epsilon^{2}+\epsilon^{2} M \log \left(1+\pi L^{2}\right)-2 \epsilon^{2}(M+1) \log L \epsilon \\
& +O\left(\epsilon^{2}\right)+O\left((L \epsilon)^{3} \log L\right)+O\left(\frac{1}{L^{4}}\right) .
\end{aligned}
$$

It is easy to see that

$$
\int_{B_{2 L \epsilon}(0)} e^{\phi_{2}} d V_{g}=O\left((L \epsilon)^{4}\right)
$$

and

$$
\int_{B_{2 L \epsilon}(0)} e^{G_{2}} d V_{g}=O\left((L \epsilon)^{4}\right) .
$$

Since $\int e^{G_{2}}=1$, we get

$$
\log \int_{\Sigma} e^{\phi_{2}}=\log \left(1-O\left((L \epsilon)^{4}\right)\right)=O\left((L \epsilon)^{4}\right) .
$$

In the end, we can deduce from (6.1), (6.2), (6.3) and (6.4) that

$$
\begin{aligned}
\Phi_{0}(\phi)= & -4 \pi-4 \pi \log \pi+2 \int G_{2} d V_{g}-\epsilon^{2}\left(\log \left(1+\pi L^{2}\right)-2 \log L \epsilon\right)(1+M) \\
& +O\left(\frac{1}{L^{4}}\right)+O\left(\epsilon^{3} \log L\right)+O\left((L \epsilon)^{4} \log L \epsilon\right)+O\left(\epsilon^{2}\right) .
\end{aligned}
$$


Let $L^{4} \epsilon^{2}=\frac{1}{\log (-\log \epsilon)}$. Then for $\epsilon$ sufficiently small we have

$$
\Phi_{0}(\phi)<-4 \pi-4 \pi \log \pi+2 \int G_{2} d V_{g}
$$

This proves our claim.

Therefore, if $\Sigma$ satisfies the condition

$$
\max _{p \in \Sigma} K(p)<2 \pi
$$

we can see that $u^{\epsilon}$ converges to $u^{0}=\left(u_{1}^{0}, u_{2}^{0}\right)$ in $H_{2}:=H^{1,2}(\Sigma) \times H^{1,2}(\Sigma)$, hence it is clear that $\Phi\left(u^{0}\right)=\inf _{u \in H_{2}} \Phi(u)$, that is, $u^{0}$ is a minimizer of $\Phi_{0}=\Phi$. This completes the proof of the main theorem.

\section{References}

[1] E. Caglioti, P.-L. Lions, C. Marchioro and M. Pulvirenti, A special class of stationary flows for two-dimensional Euler equations: a statistical mechanics description, Commun. Math. Phys. 143 (1992), 501-525.

[2] E. Caglioti, P.-L. Lions, C. Marchioro and M. Pulvirenti, A special class of stationary flows for two-dimensional Euler equations: a statistical mechanics description. Part II, Commun. Math. Phys. 174 (1995), 229-260.

[3] A. S. Y. ChAng and P. YAng, Conformal deformation of metrics on $S^{2}$, J. Differential Geom. 23 (1988), 259-296.

[4] A. S. Y. Chang and P. YAng, Prescribing Gaussian curvature on $S^{2}$, Acta Math. 159 (1987), 214-259.

[5] C.-C. CHEN and C.-S. Lin, Sharp estimates for solutions of multi-bubbles in compact Riemann surfaces, Comm. Pure Appl. Math. 55 (2002), 728-771.

[6] W. X. Chen and W. Y. Ding, Scalar curvature on $S^{2}$, Trans. Amer. Math. Soc. 303 (1987), 365-382.

[7] W. X. CHEN and C. LI, Classification of solutions of some nonlinear elliptic equations, Duke Math. J. 63 (1991) 615-622.

[8] W. Ding, J. Jost, J. Li and G. WANG, The differential equation $\Delta u=8 \pi-8 \pi h e^{u}$ on a compact Riemann surface, Asian J. Math. 1 (1997), 230-248.

[9] W. Ding, J. Jost, J. LI and G. WANG,An analysis of the two-vortex case in the ChernSimons Higgs model, Calc. Var. Partial Differential Equations 7 (1998), 87-97.

[10] W. Ding, J. Jost, J. Li and G. WANG, Multiplicity results for the two-vortex ChernSimons Higgs model on the two sphere, Comment. Math. Helv. 74 (1999), 118-142.

[11] W. Ding, J. Jost, J. Li, X. Peng and G. Wang, Self duality equations for GinzburgLandau and Seiberg-Witten type functionals with $6^{\text {th }}$ order potentials, Commun. Math. Phys. 217 (2001), 383-407.

[12] Z. DJAdli and A. MAlchiodi, Existence of conformal metrics with constant $Q$ curvature, preprint, SISSA, 70/2004/M.

[13] J. Hong, Y. KIM and P. Y. PaC, Multivortex solutions of the Abelian Chern-Simons theory, Phys. Rev. Lett. 64 (1990), 2230-2233.

[14] R. Jackiw and E. Weinberg, Self-dual Chern-Simons vortices, Phys. Rev. Lett. 64 (1990), 2234-2237.

[15] J. Jost, C.-S. Lin and G. WANG, Analytic aspects of the Toda system: II. Bubbling behavior and existence of solutions, Comm. Pure Appl. Math., to appear. 
[16] J. Jost and G. WAng, Analytic aspects of the Toda system. I. A Moser-Trudinger inequality, Comm. Pure Appl. Math. 54 (2001), 1289-1319.

[17] J. KAZDAN and F. WARNER, Curvature functions for compact 2-manifolds, Ann. of Math. 99 (1974), 14-47.

[18] Y. LI, Moser-Trudinger inequality on compact Riemannian manifolds of dimension two, J. Partial Differential Equations 14 (2001), 163-192.

[19] Y. LI, The extremal functions for Moser-Trudinger inequality on compact Riemannian manifolds, Sci. China Ser. A 48 (2005), 618-648.

[20] M. LuCiA and M. NOLASCO, Chern-Simons vortes theorey and Toda systems, J. Differential Equations 184 (2002), 443-474.

[21] M. Nolasco and G. Tarantello, Double vortex condensates in the Chern-SimonsHiggs theory, Calc. Var. Partial Differential Equations 9 (1999), 31-94.

[22] M. Nolasco and G. TARAntello, Vortex condensates for the SU(3) Chern-Simons theory, Commun. Math. Phys. 213 (2000), 599-639.

[23] M. STRUWE and G. TARANTEllo, On multivortex solutions in Chern-Simons gauge theory, Boll. Unione Mat. Ital. Sez. B Artic. Ric. Mat. (8) 1 (1998), 109-121.

[24] G. TARAntello, Multiple condensate solutions for the Chern-Simons Higgs theory, J. Math. Phys. 37 (1996), 3769-3796.

Math. Group

The abdus salam ICTP

34100 Trieste, Italy

and

Academy of Mathematics and Systems Sciences

Chinese Academy of Sciences

Beijing 100080, P. R. of China

jyli@ictp.it

Math. Group

The abdus salam ICTP

34100 Trieste, Italy

liy@ictp.trieste.it 